\title{
Investigation Performance and Mechanisms of Inverted Polymer Solar Cells by Pentacene Doped P3HT : PCBM
}

\author{
Hsin-Ying Lee and Hung-Lin Huang \\ Department of Photonics, Research Center Energy Technology and Strategy, Advanced Optoelectronic Technology Center, \\ National Cheng Kung University, Tainan 701, Taiwan \\ Correspondence should be addressed to Hsin-Ying Lee; hylee@ee.ncku.edu.tw
}

Received 24 February 2014; Accepted 13 March 2014; Published 8 April 2014

Academic Editor: Ramunas Lygaitis

Copyright (C) 2014 H.-Y. Lee and H.-L. Huang. This is an open access article distributed under the Creative Commons Attribution License, which permits unrestricted use, distribution, and reproduction in any medium, provided the original work is properly cited.

\begin{abstract}
The inverted polymer solar cells (PSCs) with pentacene-doped P3HT : PCBM absorption layers were fabricated. It was demonstrated that the pentacene doping modulated the electron mobility and the hole mobility in the resulting absorption layer. Furthermore, by varying the doping content, the optimal carrier mobility balance could be obtained. In addition, the pentacene doping led to an improvement in the crystallinity of the resulting films and made an enhancement in the light absorption, which was partly responsible for the performance improvement of the solar cells. Using the space-charge-limited current (SCLC) method, it was determined that the balanced carrier mobility $\left(\mu_{h} / \mu_{e}=1.000\right)$ was nearly achieved when a pentacene doping ratio of 0.065 by weight was doped into the P3HT: PCBM : pentacene absorption layer. Compared with the inverted PSCs without the pentacene doping, the short circuit current density and the power conversion efficiency of the inverted PSCs with the pentacene doping ratio of 0.065 were increased from $9.73 \mathrm{~mA} / \mathrm{cm}^{2}$ to $11.26 \mathrm{~mA} / \mathrm{cm}^{2}$ and from $3.39 \%$ to $4.31 \%$, respectively.
\end{abstract}

\section{Introduction}

Over the past decades, much effort has been devoted to improving energy utilization efficiency, to developing renewable energy, and to decreasing overall greenhouse gas emissions [1]. Recently, polymer solar cells (PSCs) have attracted much attention and are thought of as a potential candidate of the next generation solar cells, because they have many advantages, including low cost, flexibility, light weight, and easy fabrication $[2,3]$. However, compared with the inorganic solar cells [4-6], the PSCs suffer from two major drawbacks of a lower power conversion efficiency (PCE) and a worse stability [7]. Conventionally, the PSCs were constructed with an $\mathrm{Al}$ back cathode electrode, a poly(3,4-ethylene-dioxythiophene) : poly(styrene sulfonate) (PEDOT:PSS) hole transport layer inserted between the polymer absorption layer and the indium tin oxide (ITO) front anode electrode. Unfortunately, the oxygen could diffuse into the absorption layer through the pinholes and grain boundaries within the $\mathrm{Al}$ electrode. Consequently, the quality of the absorption layer of PSCs was degraded [8].
Furthermore, the ITO electrode was easily etched by the PEDOT: PSS [9]. These problems were responsible for the instability of the PSCs, which limited the application and commercialization of the devices. To improve the stability of the PSCs, an inverted cell structure for PSCs was previously proposed, where a high-work-function metal ( $\mathrm{Au}$ or $\mathrm{Ag}$ ) layer was used as the back contact anode electrode and the PEDOT: PSS hole transport layer was removed [10]. However, the conventional inverted PSCs still suffer from low PCE. To enhance the efficiency, many efforts have been carried out previously. For example, organic or inorganic materials were doped into the P3HT: PCBM absorption layers of the PSCs to enhance the light absorption or the carrier mobility. Various promising doping materials were previously reported, including cadmium selenide (CdSe) [11], zinc oxide ( $\mathrm{ZnO})$ [12], nanodiamonds [13], single wall carbon nanotubes (SWCNTs) [14], ferric oxide $\left(\mathrm{Fe}_{3} \mathrm{O}_{4}\right)$ [15], graphene [16], 3-hydroxyflavone (3-HF) [17], and perylene [18]. In these previous reports, the performances of PSCs were improved owing to an increase of light absorption. Consequently, the amount of the photoinduced charge carriers in the absorption 


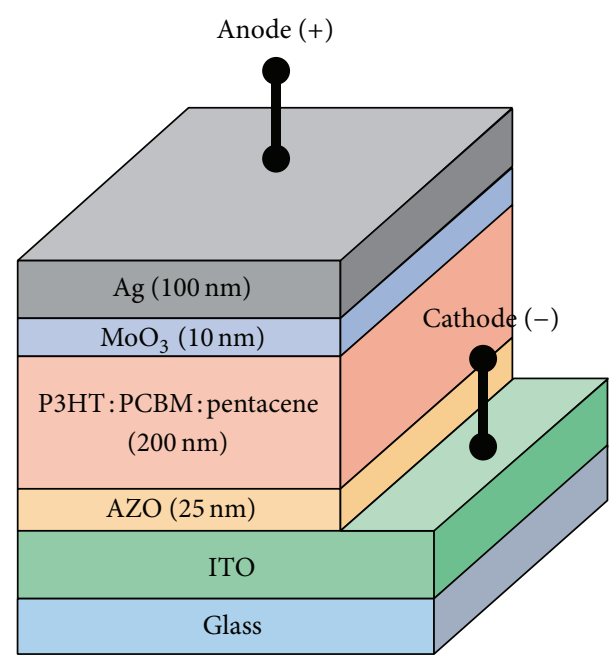

FIGURE 1: Schematic configuration of the inverted PSCs structure.

layer was increased. However, the PCE of these PSCs with the absorption layer doped with various materials was still not satisfactory, only $1.5 \% \sim 3.6 \%$. It has been pointed out that the carrier mobility mismatching in the absorption layer was one of the main reasons for the low PCE [19]. The balanced carrier mobility could decrease the carrier recombination in the absorption layer and hence increase the photocurrent of the resulting solar cells [20].

Recently, many efforts have been devoted to balancing the carrier mobility in the absorption layer. It was reported that the carrier mobility could be modulated by doping pentacene into the absorption layer of the conventional PSCs and the performances of the resulting devices were improved [21]. In order to further improve the performance of the PSCs, in this work, the inverted PSCs with pentacene-doped absorption layer were fabricated and investigated. To clearly identify the electron mobility and the hole mobility in the absorption layers, the electron-only devices and the holeonly devices with the corresponding absorption layers were analyzed, respectively, using the space-charge-limited current (SCLC) method. By varying pentacene doping content in the absorption layer, the optimal mobility balance condition was obtained. It is clarified that the PCE of the inverted PSCs was enhanced by properly balancing the carrier mobility in the absorption layer.

\section{Experiments}

Figure 1 shows the schematic configuration of the inverted polymer solar cells (PSCs). The $25 \mathrm{~nm}$ thick Al-doped $\mathrm{ZnO}$ (AZO) film was deposited on the ITO-coated glass substrate using a magnetron radio-frequency (RF) sputtering system. The AZO film worked as the electron transportation and hole blocking layer. The mixed solution of poly(3-hexylthiophene) (P3HT), (6,6)-phenyl- $\mathrm{C}_{61}$-butyric acid methyl ester (PCBM), and pentacene with given mixing ratio in the 1,2-dichlorobenzene (DCB) was then spread on the AZO film using a spin-coating technique to form a
P3HT: PCBM : pentacene absorption layer of the inverted PSCs. The thickness of the absorption layer was $200 \mathrm{~nm}$. Subsequently, the deposited absorption layer was annealed in a nitrogen glove box at $110^{\circ} \mathrm{C}$ for 20 minutes. Finally, the $10 \mathrm{~nm}$ thick $\mathrm{MoO}_{3}$ layer and the $100 \mathrm{~nm}$ thick $\mathrm{Ag}$ layer were subsequently deposited on the absorption layer as the anode electrode of the inverted PSCs using a thermal evaporator. The absorption area of the inverted PSCs was about $4 \mathrm{~mm}^{2}$. Thus fabricated inverted PSCs with various pentacene doping ratios $(0,0.05,0.06,0.065$, and 0.07 by weight) in the P3HT: PCBM (1:0.8) absorption layers were, respectively, named solar cells A, B, C, D, and E, hereafter. For estimating the hole mobility and the electron mobility in the absorption layer, the hole-only devices of $\mathrm{Au} / \mathrm{P} 3 \mathrm{HT}: \mathrm{PCBM}$ : pentacene/ $\mathrm{MoO}_{3} / \mathrm{Ag} \quad(100 /$ 200/10/100 nm) and the electron-only devices of ITO/AZO/ P3HT : PCBM : pentacene/Al (300/25/200/100 nm) were fabricated. In this work, ten batches, each batch had six devices, of the electron-only devices, the hole-only devices, and the inverted PSCs were fabricated and measured.

The space-charge-limited current (SCLC) method was used to estimate the hole mobility and the electron mobility in the absorption layer for the hole-only devices and the electron-only devices, respectively. The crystallinity and surface morphology of the absorption layers with various pentacene doping contents were measured using $\mathrm{X}$ ray diffraction (XRD) and atomic force microscopy (AFM), respectively. The current density versus voltage $(J-V)$ characteristics of the inverted PSCs were measured at room temperature using a $J-V$ curve tracer (Keithley 2400) with an AM $1.5 \mathrm{G}$ solar simulator $\left(100 \mathrm{~mW} / \mathrm{cm}^{2}\right)$. The external quantum efficiency (EQE) was measured using a chopped calibrated light beam from a xenon lamp combined with a lock-in amplifier. The absorption and the diffuse reflection spectra of the absorption layer with various pentacene doping contents and the resulting cells were measured using an UVVis spectrometer (Hitachi, U4100).

\section{Experimental Results and Discussion}

The SCLC method was used to estimate the electron mobility $\left(\mu_{e}\right)$ and the hole mobility $\left(\mu_{h}\right)$ in various absorption layers by using the corresponding electron-only and hole-only devices, respectively. The dark current density-voltage characteristics of the electron-only devices and the hole-only devices with the absorption layers of various pentacene doping contents were shown in Figure 2. The electron mobility of the electrononly devices and the hole mobility of the hole-only devices were estimated by Mott-Gurney law equation shown as follows [22]:

$$
J=\frac{9}{8} \varepsilon_{0} \varepsilon_{r} \mu \frac{V^{2}}{d^{3}},
$$

where $J$ is the dark current density, $\varepsilon_{0} \varepsilon_{r}$ is the permittivity of the P3HT: PCBM : pentacene absorption layer, which was estimated to be (average value \pm standard deviation) (4.50 \pm $0.02) \times 10^{-11} \mathrm{~F} / \mathrm{m}$ from the capacitance-voltage measurement results, $\mu$ is the carrier mobility, $V$ is the applied voltage, and 


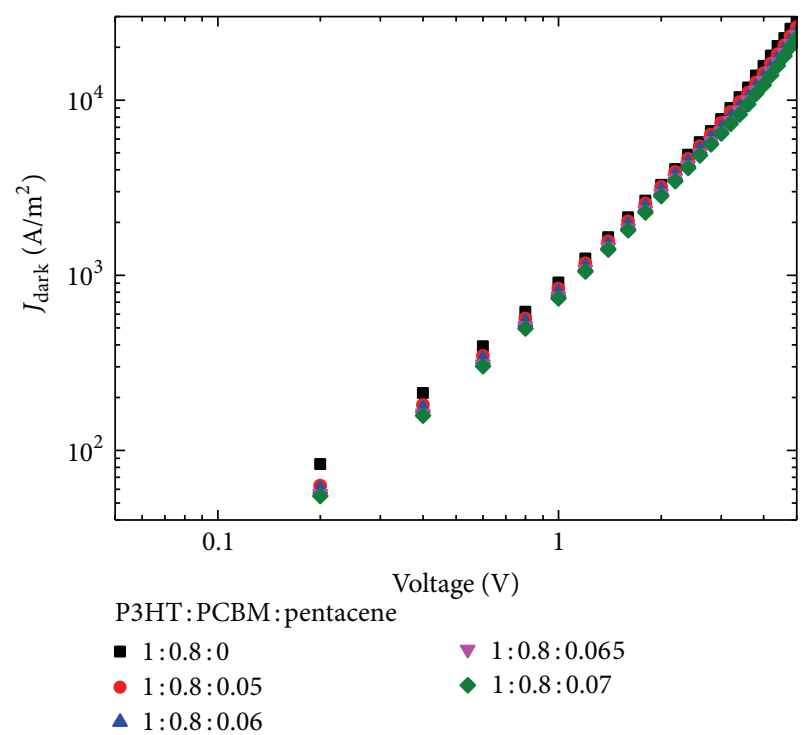

(a)

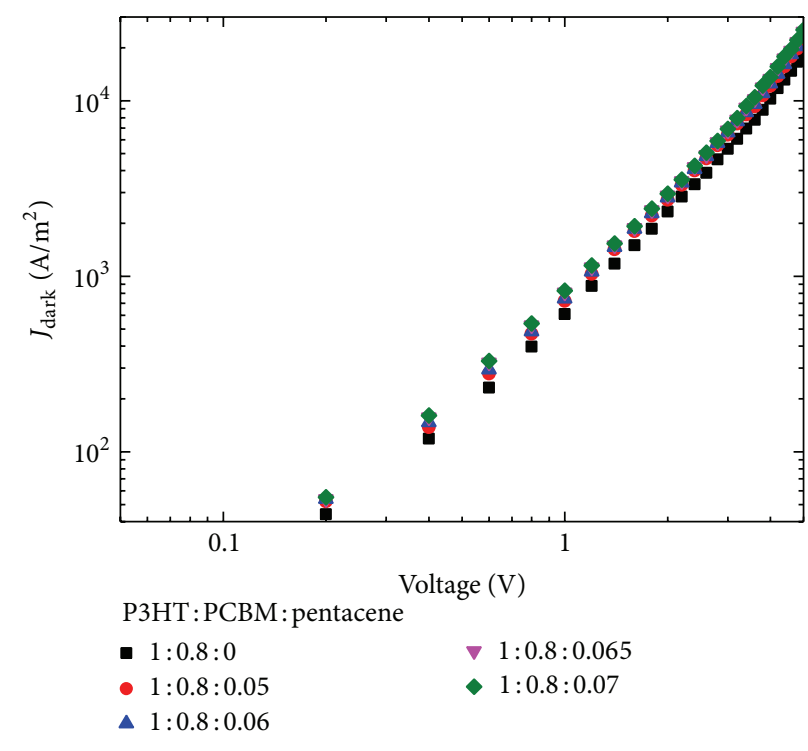

(b)

Figure 2: The dark current densities for (a) electron-only devices and (b) hole-only devices with the absorption layer of various pentacene doping contents.

TABLE 1: The hole mobility and electron mobility of the hole-only devices and the electron-only devices with various pentacene doping contents.

\begin{tabular}{lccc}
\hline P3HT : PCBM : pentacene & $\begin{array}{c}\text { Hole mobility } \\
\mu_{h}\left(10^{-3} \mathrm{~cm}^{2} / \mathrm{Vs}\right)\end{array}$ & $\begin{array}{c}\text { Electron } \\
\text { mobility } \\
\mu_{e}\left(10^{-3} \mathrm{~cm}^{2} / \mathrm{Vs}\right)\end{array}$ & $\mu_{h} / \mu_{e}$ \\
\hline $1: 0.8: 0$ & $0.94 \pm 0.01$ & $1.37 \pm 0.01$ & $0.686 \pm 0.002$ \\
$1: 0.8: 0.05$ & $1.09 \pm 0.02$ & $1.29 \pm 0.02$ & $0.845 \pm 0.002$ \\
$1: 0.8: 0.06$ & $1.13 \pm 0.01$ & $1.21 \pm 0.01$ & $0.934 \pm 0.001$ \\
$1: 0.8: 0.065$ & $1.16 \pm 0.01$ & $1.16 \pm 0.01$ & $1.000 \pm 0.001$ \\
$1: 0.8: 0.07$ & $1.18 \pm 0.02$ & $1.13 \pm 0.02$ & $1.044 \pm 0.001$ \\
\hline
\end{tabular}

$d$ is the thickness of the absorption layer of the devices. This equation could be also rewritten and shown as follows:

$$
\log (J)=\log \left(\frac{9 \varepsilon_{0} \varepsilon_{r} \mu}{8 d^{3}}\right)+2 \log (V)
$$

To conform the Mott-Gurney law, the slope of $\log (J)-\log (V)$ curve for the electron-only devices and the hole-only devices should be 2 . In this work, the applied voltage of $1.7 \mathrm{~V}$ matched in Mott-Gurney law was chosen to estimate the hole mobility of the hole-only devices and the electron mobility of the electron-only devices. Thus, the electric field $(E)$ of all devices estimated by the formula of $E=V / d$, where $V$ of $1.7 \mathrm{~V}$ is the applied voltage and $d$ of $200 \mathrm{~nm}$ is the thickness of the absorption layer, was $8.5 \times 10^{4} \mathrm{~V} / \mathrm{cm}$. The resulting hole mobility and the electron mobility in the absorption layers with various pentacene doping contents are listed in Table 1. It can be seen that the hole mobility of the absorption layer increased and the electron mobility of the absorption layer decreased with increasing the pentacene doping content. In particular, for the P3HT: PCBM absorption layer with pentacene doping ratio of 0.065 , the hole mobility, compared with the P3HT: PCBM absorption layer, was increased from $(0.94 \pm 0.01) \times 10^{-3} \mathrm{~cm}^{2} / \mathrm{Vs}$ to $(1.16 \pm 0.01) \times 10^{-3} \mathrm{~cm}^{2} / \mathrm{Vs}$. Contrarily, the electron mobility in the absorption layer was decreased from $(1.37 \pm 0.01) \times 10^{-3} \mathrm{~cm}^{2} / \mathrm{Vs}$ to $(1.16 \pm$ $0.01) \times 10^{-3} \mathrm{~cm}^{2} / \mathrm{Vs}$. The opposite variation of the electron mobility and the hole mobility with pentacene doping content indicated that the ratio of the hole mobility and the electron mobility was accordingly modulated. As seen from the results listed in Table 1, the carrier mobility ratio varied with the pentacene content and, in particular, balanced carrier mobility of $1.000 \pm 0.001$ was obtained in the absorption layer with a pentacene doping ratio of 0.065 by weight.

The variation of the carrier mobility upon pentacene doping can be understood based on the photovoltaic process in the polymer solar cells (PSCs) described below. Figure 3 shows the carrier transport process as well as the energy level diagram of the component materials in the inverted PSCs with $\mathrm{P} 3 \mathrm{HT}$ : $\mathrm{PCBM}$ : pentacene absorption layer. In the process, the generation and transport of the carriers in the absorption layer played the most important role and were illustrated in detail in Figure 4. As seen from Figure 4, 


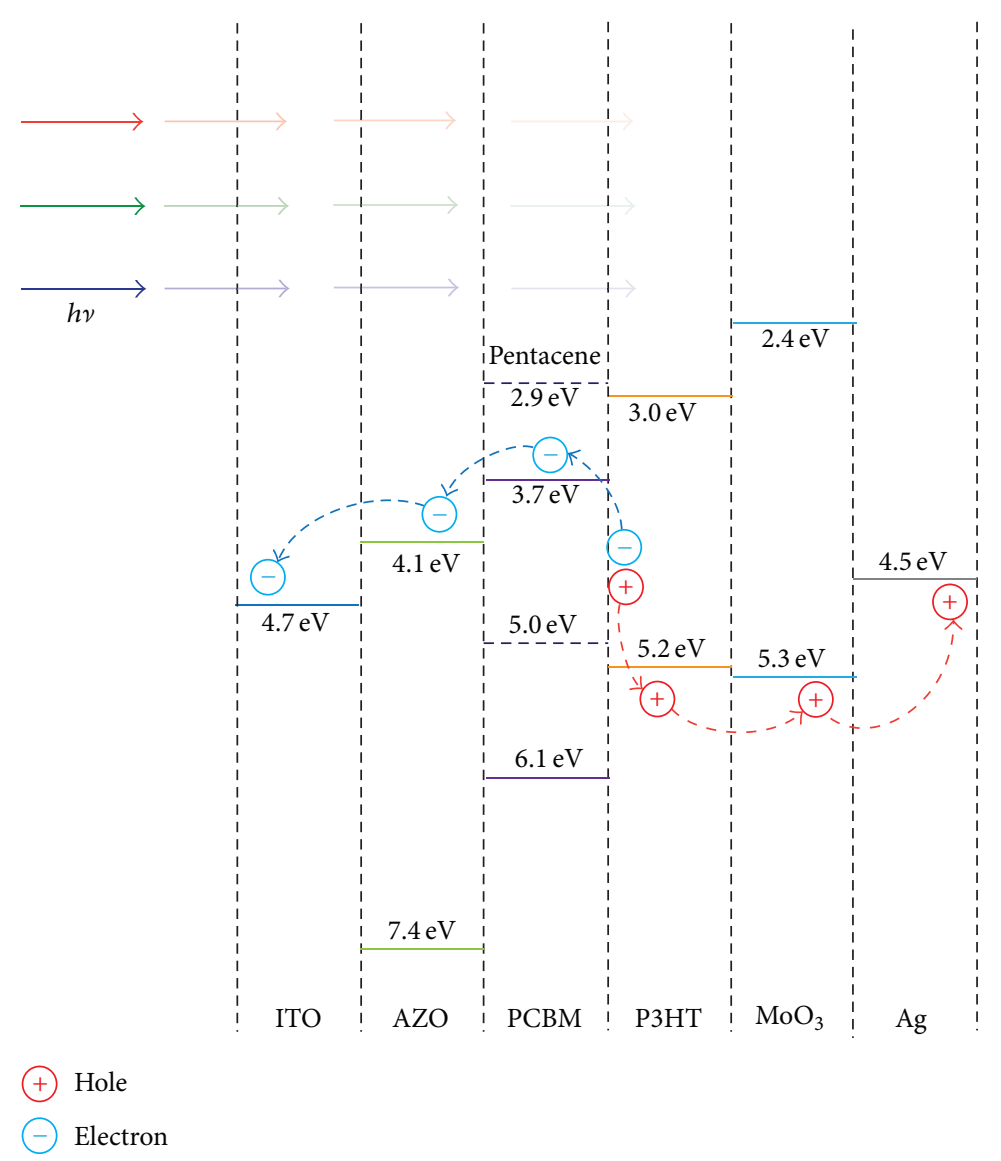

FIGURE 3: Schematic diagram of energy levels for inverted PSCs with pentacene-doped absorption layer.

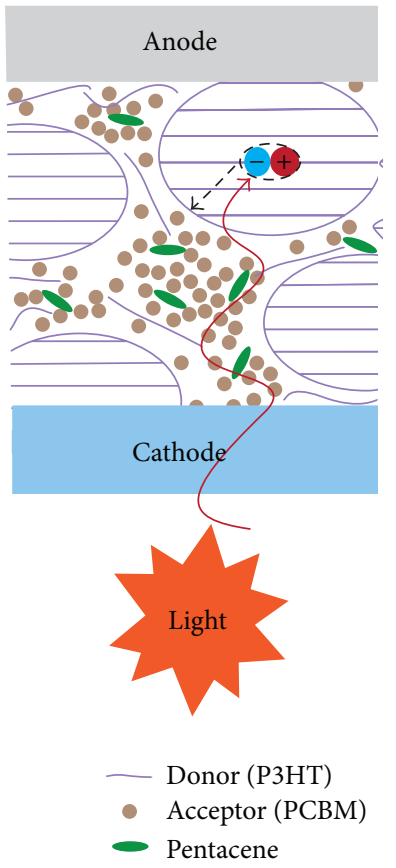

(a)

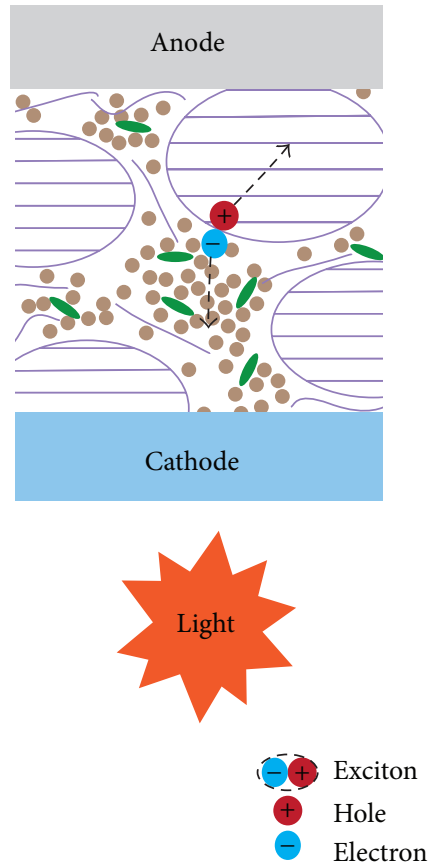

(b)

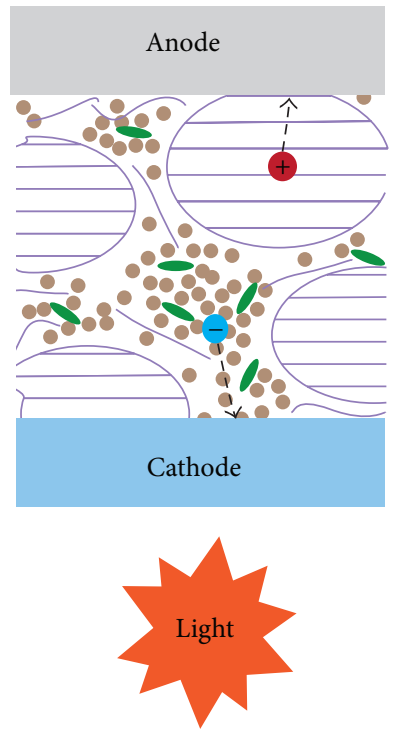

(c)

FIGURE 4: Schematics of photocurrent generation in P3HT: PCBM : pentacene system. 


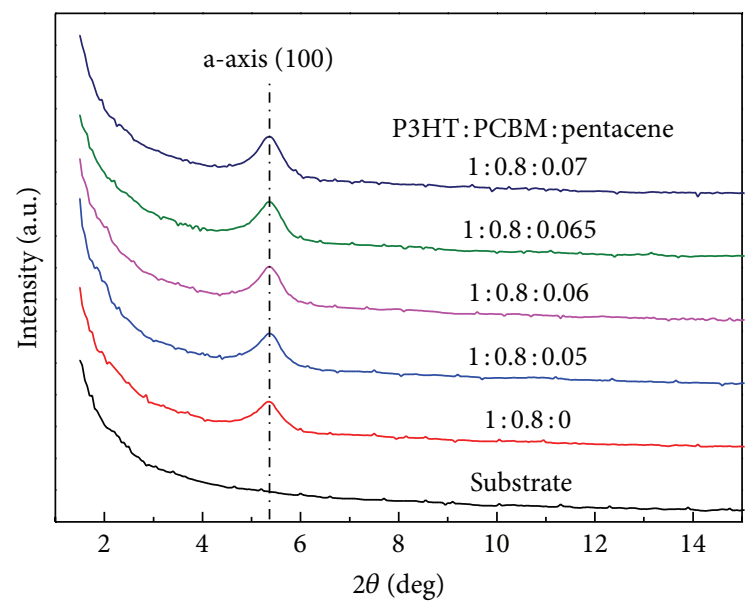

FIGURE 5: XRD spectra of the absorption layers with various pentacene doping contents.

the domains of the donor material were separated in the acceptor materials. When the incident light was absorbed by the donor material, electron-hole excitons were generated in P3HT, as shown in Figure 4(a). The excitons diffused to the interface between the donor and acceptor materials, as shown in both Figures 3 and 4(b). At the interface, the excitons were dissociated and the resulting electrons are transited into the electronegative acceptor materials, whereas the resulting holes remained in the P3HT, as shown in Figure 4(c). Afterwards, the resultant electrons and holes are transported towards the corresponding electrodes [23] as shown in Figure 3. However, due to the energy difference between LUMO of pentacene (electron affinity, $2.9 \mathrm{eV}$ ) [24] and the LUMO of PCBM (electron affinity, $3.7 \mathrm{eV}$ ) [25], as shown in Figure 3, the addition of pentacene doped into the absorption layer obstructed the electron transportation and decreased the electron mobility in the absorption layer. Consequently, the electron mobility in the absorption layer decreased with an increase of the pentacene doping content. On the other hand, the hole mobility enhancement could be attributed to the improvement in the crystallinity of the $\mathrm{P} 3 \mathrm{HT}$, as reported previously [26]. To demonstrate this phenomenon, the crystallinity analyses of the absorption layers with various pentacene doping contents were carried out using XRD and the results are shown in Figure 5. As shown in Figure 5, the XRD spectra of all the deposited absorption layers exhibited a (100) diffraction peak of the a-axis orientation of P3HT [27]. Moreover, the intensity of the diffraction peak increased with an increase of the pentacene doping content. These results indicated that the crystallinity of the $\mathrm{P} 3 \mathrm{HT}$ in the absorption layer was improved by doping the pentacene. This phenomenon indicated that the hole mobility enhancement could be attributed to the enhancement in the crystallinity, induced by pentacene doping, of the $\mathrm{P} 3 \mathrm{HT}$ in the absorption layer.

Except the mobility balance in the absorption layer, changes in the other properties of the absorption layer upon pentacene doping might affect the performances of the resulting solar cells. Figure 6 shows the absorption spectra, in

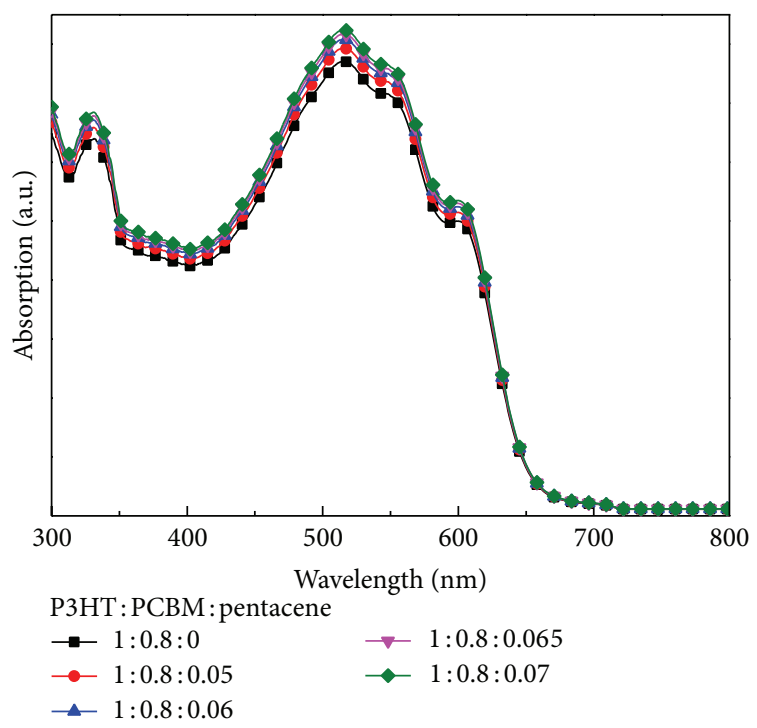

FIGURE 6: The UV-Vis absorption spectra of the absorption layers with various pentacene doping contents.

which the wavelength ranged from $300 \mathrm{~nm}$ to $800 \mathrm{~nm}$, of the absorption layers with various pentacene doping contents. As shown in Figure 6, the absorption of the absorption layers increased with an increase of the pentacene doping weight ratio. The absorption enhancement is obviously favorable to the solar cell performance. In general, the absorptivity of the polymer is larger as the electric field of the incident light is aligned parallel to the orientation of the polymer main chains [28]. In other words, the improvement of the P3HT crystallinity enhances its absorption for the light incident perpendicularly to the main chains of the crystalline P3HT. In our case, as observed above by XRD analysis, the intensity of the (100) diffraction peak for the P3HT, which corresponded to an alignment of P3HT main chain parallelly to the substrate [27], increased with an increase of the pentacene doping content. It implied an improvement of P3HT crystallization with its main chain parallel to the substrate. Therefore, according to the previous observation [28], the absorptivity enhancement of the absorption layer was attributed to the crystallinity improvement of the $\mathrm{P} 3 \mathrm{HT}$ in the pentacene-doped absorption layer. Furthermore, Figure 7 shows the surface morphologies of the absorption layers with various pentacene doping contents. The root mean square $(\mathrm{rms})$ surface roughness of the P3HT: PCBM : pentacene $(1: 0.8: 0,1: 0.8: 0.05,1: 0.8: 0.06$, $1: 0.8: 0.065$, and $1: 0.8: 0.07)$ absorption layer was $1.94 \mathrm{~nm}$, $2.93 \mathrm{~nm}, 3.23 \mathrm{~nm}, 3.69 \mathrm{~nm}$, and $3.93 \mathrm{~nm}$, respectively. It could be found that the surface of the P3HT:PCBM:pentacene absorption layer was more roughened with an increase of the pentacene doping content. It was reported previously that the larger roughened surface of the P3HT : PCBM films indicated that the P3HT had better crystallinity $[13,29]$. Based on this observation, it could also be deducted that the crystallinity of the P3HT : PCBM : pentacene absorption layer was improved with an increase of the pentacene content, which was consistent with the above-mentioned XRD measurement results. 


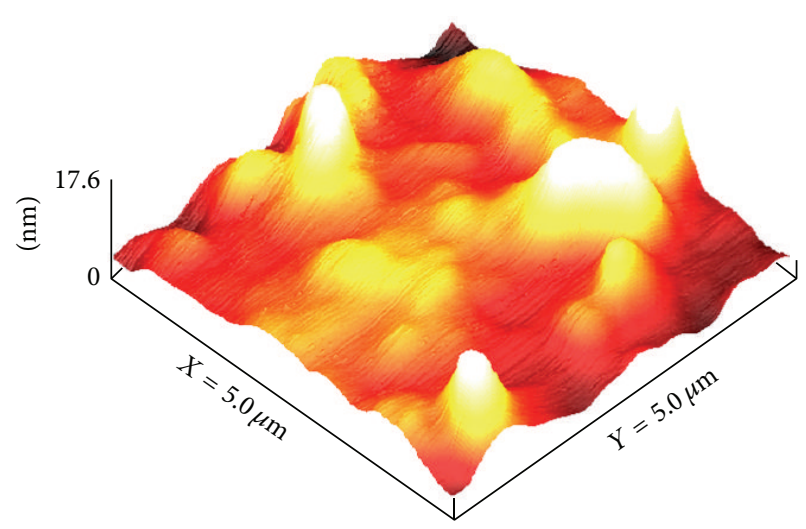

rms roughness $=1.94 \mathrm{~nm}$

(a)

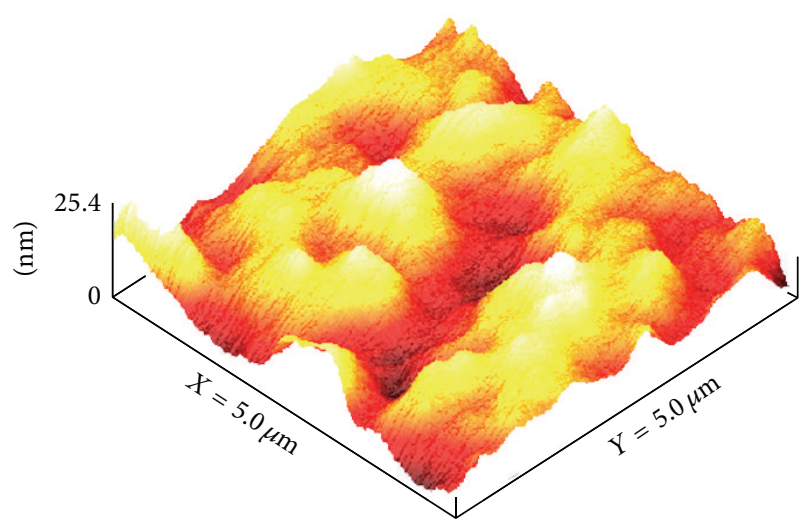

rms roughness $=3.23 \mathrm{~nm}$

(c)

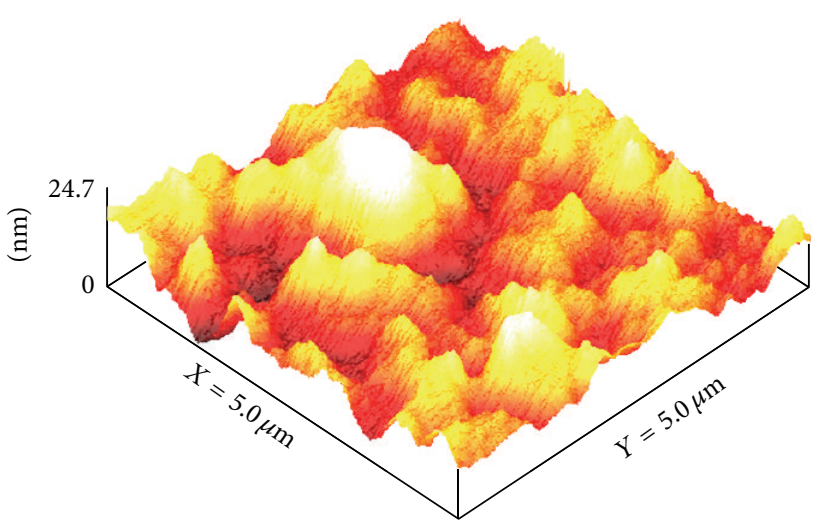

rms roughness $=2.93 \mathrm{~nm}$

(b)

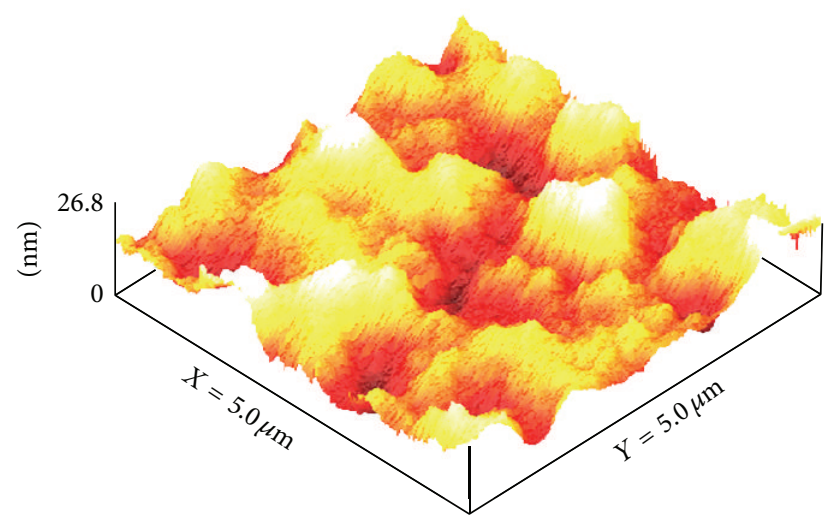

rms roughness $=3.69 \mathrm{~nm}$

(d)

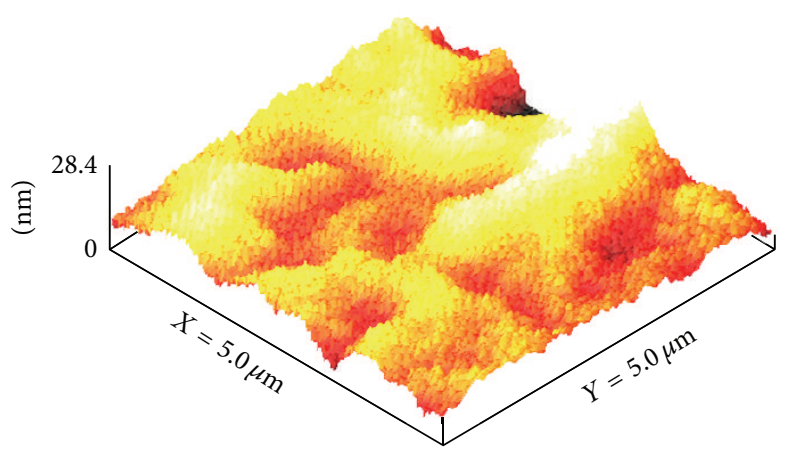

$\mathrm{rms}$ roughness $=3.93 \mathrm{~nm}$

(e)

FIGURE 7: AFM images and rms surface roughness of the absorption layers with the pentacene doping ratio of (a) 0 , (b) 0.05 , (c) 0.06 , (d) 0.065 , and (e) 0.07 by weight.

Moreover, the increased surface roughness could enhance the light utilization via internal reflection and scattering at the roughened surface, which was also beneficial to the exciton production. To clearly demonstrate this feature, the reflectivity spectra of the $\mathrm{P} 3 \mathrm{HT}$ : $\mathrm{PCBM}$ : pentacene inverted PSCs with various pentacene doped absorption layers were measured and the results are shown in Figure 8. As shown in Figure 8, the reflectivity of the inverted PSCs was slightly decreased with an increase of the pentacene doping content, which implied that the diffused reflection light from the roughened surface was more effectively absorbed by absorption layer. Besides, the roughened surface increased the contact area between the polymer film and the metal anode. Therefore, the photocurrent of the PSCs could be increased [30]. As demonstrated in the above discussion, pentacene doping improved the crystallinity of the $\mathrm{P} 3 \mathrm{HT}$ in 


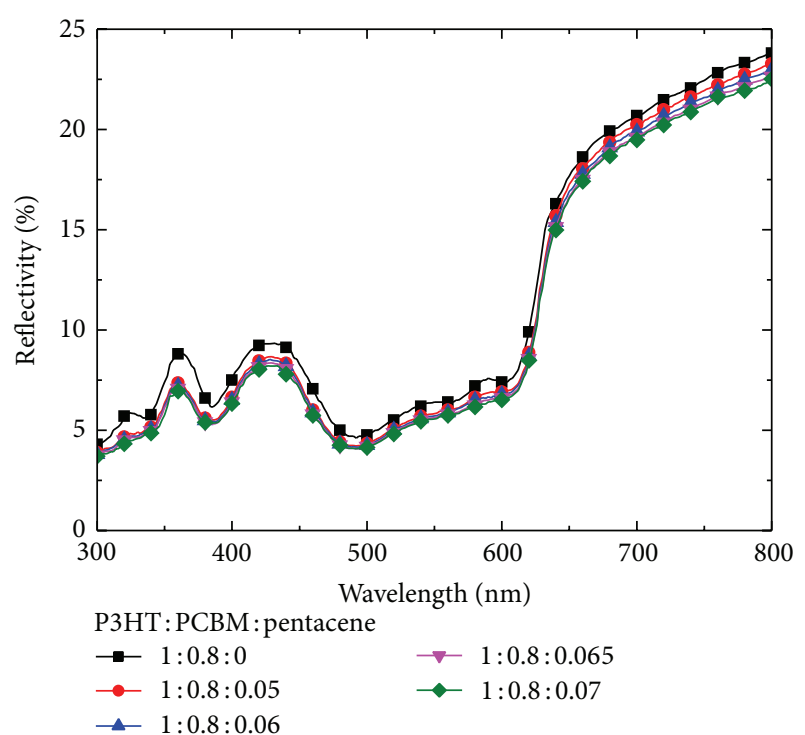

FIGURE 8: The reflectivity of the inverted PSCs with various pentacene-doped absorption layers.

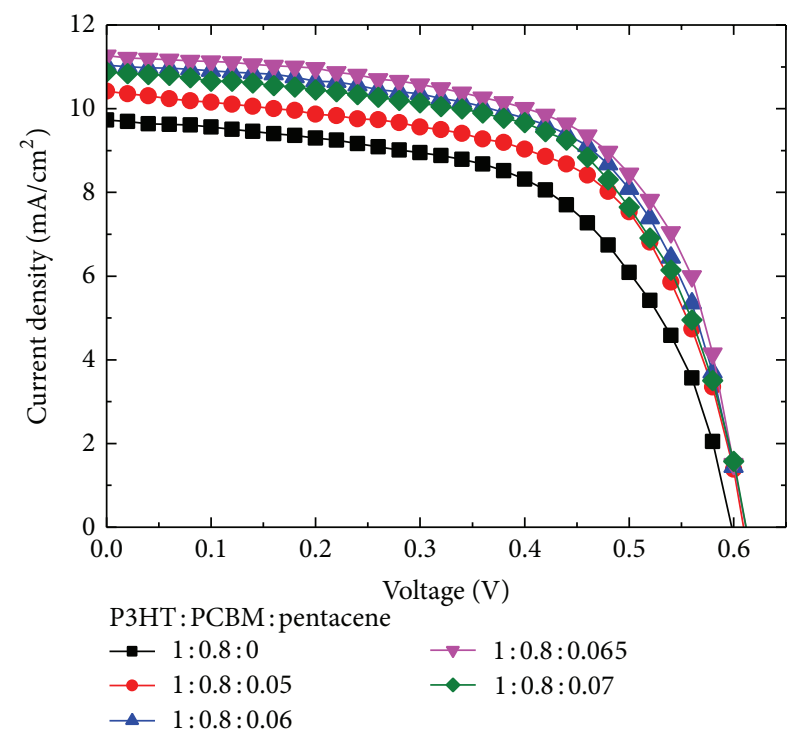

FIGURE 9: The current density-voltage characteristics of the inverted PSCs (solar cells A, B, C, D, and E).

the absorption layer, which in turn caused changes in the carrier mobility, absorption, and surface roughness of the absorption layer. All of these changes affected the performances of the resulting devices.

Figure 9 shows the $J-V$ characteristics of the inverted PSCs with absorption layer of various pentacene doping contents. The photovoltaic characteristics of solar cells A, $\mathrm{B}, \mathrm{C}, \mathrm{D}$, and $\mathrm{E}$, including short circuit current density $\left(J_{\mathrm{sc}}\right)$, open circuit voltage $\left(V_{\mathrm{oc}}\right)$, fill factor $(\mathrm{FF})$, and power conversion efficiency (PCE), were derived from the measured $J-V$ characteristics and the results are listed in Table 2. It was found that only the open circuit voltage $V_{\text {oc }}$ was kept nearly the same for all the fabricated devices. This fact could

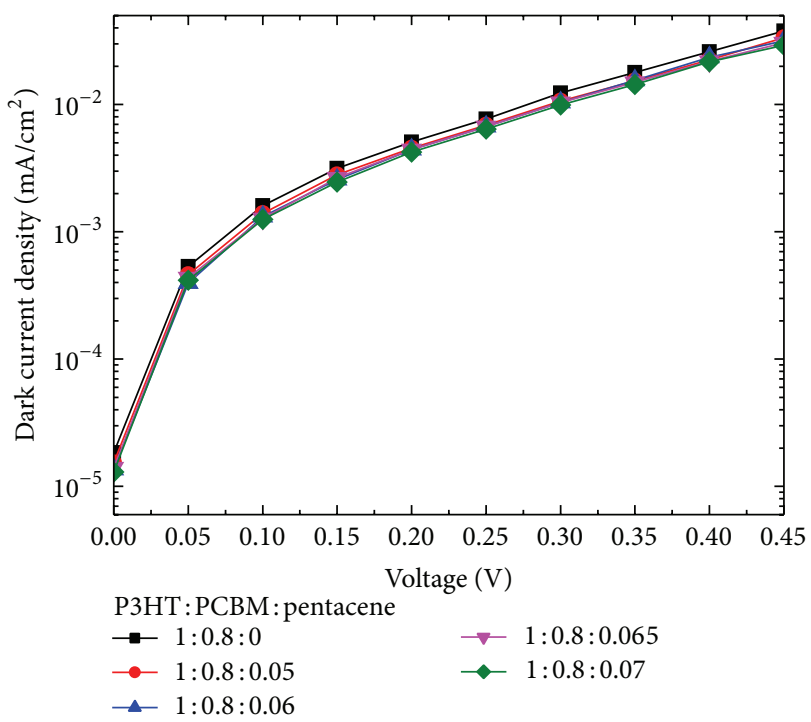

FIgURE 10: Dark current density-voltage characteristics of the inverted PSCs with various pentacene-doped absorption layers.

be evidenced from the relationship of $V_{\text {oc }}$ with the reverse saturation current density $J_{0}[31]$ :

$$
V_{\mathrm{oc}}=\frac{n K T}{q} \ln \left[\left(\frac{J_{\mathrm{ph}}}{J_{0}}\right)+1\right],
$$

where $n$ is the ideality factor, $q$ is the electron charge, $K$ is Boltzmann's constant, $T$ is the absolute temperature, $J_{\mathrm{ph}}$ is the photocurrent density, and $J_{0}$ is deduced by extrapolating the linear regions of the dark current density-voltage curve (Figure 10) to $V=0$. These devices with various pentacene doping contents exhibited similar dark current density and correspondingly had the similar $V_{\text {oc }}$. It could be seen that the performances, other than $V_{\text {oc }}$, of the inverted PSCs were improved by doping pentacene of a low ratio into the absorption layer and were optimized when the weight ratio of pentacene was 0.065 (solar cell D). For this optimized cell, the $J_{\mathrm{sc}}$ and PCE were $11.26 \pm 0.04 \mathrm{~mA} / \mathrm{cm}^{2}$ and $4.31 \pm$ $0.03 \%$, respectively, which were obviously better than those of $9.73 \pm 0.03 \mathrm{~mA} / \mathrm{cm}^{2}$ and $3.39 \pm 0.02 \%$ for solar cell A. To further investigate the variation of the above-mentioned solar cell performances, the external quantum efficiency (EQE) of the inverted PSCs with various pentacene doping contents was measured in the wavelength ranged from $300 \mathrm{~nm}$ to $800 \mathrm{~nm}$. The results, as shown in Figure 11, exhibited a similar variation as the pentacene content varied. For example, at the wavelength of $515 \mathrm{~nm}$, the EQE of solar cells A, B, C, D, and $\mathrm{E}$ was $51.4 \pm 0.1 \%, 55.0 \pm 0.1 \%, 57.4 \pm 0.1 \%, 59.5 \pm 0.1 \%$, and $58.3 \pm 0.1 \%$, respectively, in which solar cell $\mathrm{D}$ was the best one.

The results discussed above indicated that the solar cell efficiency (EQE or PCE) was increased with an increase of the pentacene doping content in the absorption layer when the doping ratio was low and reached the maximum at the doping ratio of 0.065 . When the pentacene doping ratio was further increased to 0.07 (solar cell E), the EQE was 
TABLE 2: The parameters of the inverted polymer solar cells with various pentacene doping contents.

\begin{tabular}{|c|c|c|c|c|c|}
\hline Solar cell & P3HT: PCBM : pentacene & $J_{\mathrm{sc}}\left(\mathrm{mA} / \mathrm{cm}^{2}\right)$ & $V_{\mathrm{oc}}(\mathrm{V})$ & $\mathrm{FF}(\%)$ & PCE (\%) \\
\hline A & $1: 0.8: 0$ & $9.73 \pm 0.03$ & $0.598 \pm 0.001$ & $58.3 \pm 0.1$ & $3.39 \pm 0.02$ \\
\hline B & $1: 0.8: 0.05$ & $10.41 \pm 0.03$ & $0.609 \pm 0.001$ & $60.1 \pm 0.2$ & $3.87 \pm 0.03$ \\
\hline $\mathrm{C}$ & $1: 0.8: 0.06$ & $11.04 \pm 0.03$ & $0.610 \pm 0.001$ & $62.2 \pm 0.2$ & $4.19 \pm 0.03$ \\
\hline $\mathrm{D}$ & $1: 0.8: 0.065$ & $11.26 \pm 0.04$ & $0.611 \pm 0.001$ & $62.6 \pm 0.1$ & $4.31 \pm 0.03$ \\
\hline $\mathrm{E}$ & $1: 0.8: 0.07$ & $10.87 \pm 0.04$ & $0.611 \pm 0.001$ & $61.3 \pm 0.1$ & $4.07 \pm 0.03$ \\
\hline
\end{tabular}

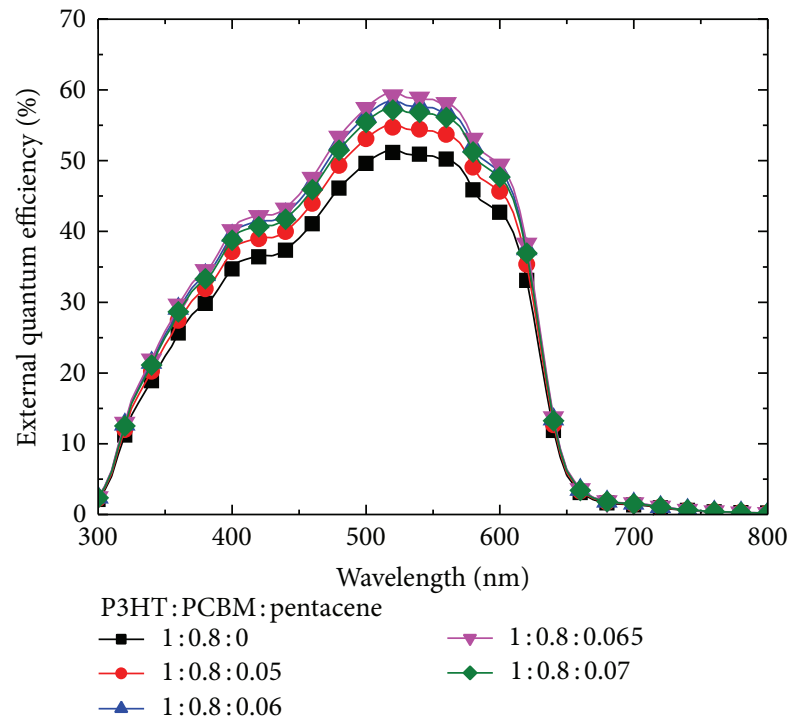

FIGURE 11: External quantum efficiency spectra of the inverted PSCs (solar cells A, B, C, D, and E).

degraded. The efficiency improvement at lower pentacene doping ratio could be attributed to both the enhancement in the absorptivity of the absorption layer and the improvement in the carrier mobility balance. Obviously, the efficiency degradation at higher doping ratio could not be ascribed to the change in the absorptivity of the absorption layer. As mentioned above, the absorptivity increased monotonically with the pentacene doping ratio, which tended to enhance the solar cell efficiency. However, it was noticed that the efficiency of the solar cell varied in a similar way as the carrier mobility ratio did (see Table 1). The optimal efficiency was achieved at the same pentacene doping ratio when the mobility in the absorption layer was properly balanced. At a higher doping ratio, say 0.07 , the EQE degraded, while the carrier mobility ratio was increased to $1.044 \pm 0.001$, departing obviously from the balance. This unbalanced mobility resulted in an accumulation of low mobility carriers, causing an increase in carrier recombination and a decrease in PCE and EQE. This kind of correlation between EQE and the mobility balance implied that the carrier mobility balance played an important role in the efficiency variation.

\section{Conclusion}

In summary, the inverted PSCs with various pentacenedoped absorption layers were fabricated. Using the SCLC method to measure and estimate the hole mobility and the electron mobility in the resulting absorption layer with various pentacene doping contents, it was revealed that the carrier mobility in the absorption layer could be modulated by doping various pentacene contents. In particular, the required carrier mobility balance $\left(\mu_{h} / \mu_{e}=1.000\right)$ was obtained in the P3HT: PCBM absorption layer with the pentacene doping ratio of 0.065 . Using the absorption layer with the balanced carrier mobility could reduce the carrier recombination in the absorption layers and hence enhance the photocurrent of the resulting inverted PSCs. Moreover, more electron-hole excitons were generated in the pentacenedoped absorption layer due to larger absorptivity and larger surface roughness, which provided additional contribution to the performance improvement. The maximum PCE of $4.31 \pm 0.03 \%$ was obtained for the inverted PSCs with the pentacene doping ratio of 0.065 in the absorption layer.

\section{Conflict of Interests}

The authors declare that there is no conflict of interests regarding the publication of this paper.

\section{Acknowledgment}

The authors gratefully acknowledge the support from the Advanced Optoelectronic Technology Center and Research Center Energy Technology and Strategy of the National Cheng Kung University, the Bureau of Energy, Ministry of Economic Affairs of the Republic of China under Contract no. TDPA 101-EC-17-A-08-S1-204 and the National Science Council of Taiwan under Contract no. NSC 102-3113-P-492002 and NSC 101-2628-E-006-017-MY3.

\section{References}

[1] G. Najafi, B. Ghobadian, T. Tavakoli, D. R. Buttsworth, T. F. Yusaf, and M. Faizollahnejad, "Performance and exhaust emissions of a gasoline engine with ethanol blended gasoline fuels using artificial neural network," Applied Energy, vol. 86, no. 5, pp. 630-639, 2009.

[2] L. Li, D. L. Jacobs, Y. Che et al., "Poly(3-hexylthiophene) nanofiber networks for enhancing the morphology stability of polymer solar cells," Organic Electronics, vol. 14, no. 5, pp. 13831390, 2013.

[3] L. Qiao, D. Wang, L. Zuo et al., "Localized surface plasmon resonance enhanced organic solar cell with gold nanospheres," Applied Energy, vol. 88, no. 3, pp. 848-852, 2011. 


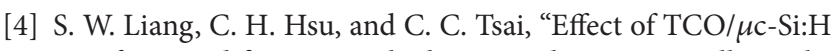
interface modification on hydrogenated microcrystalline silicon thin-film solar cells," International Journal of Photoenergy, vol. 2013, Article ID 756084, 6 pages, 2013.

[5] D. M. Chen, Z. C. Liang, L. Zhuang, Y. H. Lin, and H. Shen, "A novel method to achieve selective emitter for silicon solar cell using low cost pattern-able a-Si thin films as the semitransparent phosphorus diffusion barrier," Applied Energy, vol. 92, pp. 315-321, 2012.

[6] C. Y. Tseng and C. T. Lee, "Mechanisms of $\left(\mathrm{NH}_{4}\right)_{2} \mathrm{~S}_{x}$-treated III-V compound triple-junction solar cells incorporating with hybrid electrode," Applied Physics Letters, vol. 101, no. 3, Article ID 033902, 4 pages, 2012.

[7] Y. Sun, J. H. Seo, C. J. Takacs, J. Seifter, and A. J. Heeger, "Inverted polymer solar cells integrated with a low-temperature-annealed sol-gel-derived $\mathrm{ZnO}$ film as an electron transport layer," Advanced Materials, vol. 23, no. 14, pp. 1679-1683, 2011.

[8] J. Li, X. Huang, J. Yuan, K. Lu, W. Yue, and W. Ma, "A new alcohol-soluble electron-transporting molecule for efficient inverted polymer solar cells," Organic Electronics, vol. 14, no. 9, pp. 2167-2171, 2013.

[9] Y. M. Chang and C. Y. Leu, "Solvent extraction induced nanoporous zinc oxide as an electron transport layer for inverted polymer solar cells," Organic Electronics, vol. 13, no. 12, pp. 29912996, 2012.

[10] H. Y. Lee, H. L. Huang, and C. T. Lee, "Performance enhancement of inverted polymer solar cells using roughened Al-doped ZnO nanorod array," Applied Physics Express, vol. 5, no. 12, Article ID 122302, 3 pages, 2012.

[11] E. K. Park, H. Fu, M. Choi, W. Luan, and Y. S. Kim, "Effects ligand-exchanged cadmium selenide nanoparticles on the performance of P3HT:PCBM:CdSe ternary system solar cells," Bulletin of the Korean Chemical Society, vol. 34, no. 8, pp. 23212324, 2013.

[12] V. Kruefu, E. Peterson, C. Khantha, C. Siriwong, S. Phanichphant, and D. L. Carroll, "Flame-made niobium doped zinc oxide nanoparticles in bulk heterojunction solar cells," Applied Physics Letters, vol. 97, no. 5, Article ID 053302, 3 pages, 2010.

[13] Y. J. Hsiao, T. H. Fang, L. W. Ji, Y. C. Lee, and B. T. Dai, "Nanodiamonds embedded in P3HT:PCBM for enhancing efficiency and reliability of hybrid photovoltaics," Electrochemical and SolidState Letters, vol. 15, no. 4, pp. K27-K30, 2012.

[14] E. Kymakis, N. Kornilios, and E. Koudoumas, "Carbon nanotube doping of P3HT:PCBM photovoltaic devices," Journal of Physics D: Applied Physics, vol. 41, no. 16, Article ID 165110, 5 pages, 2008.

[15] W. Zhang, Y. Xu, H. Wang, C. Xu, and S. Yang, " $\mathrm{Fe}_{3} \mathrm{O}_{4}$ nanoparticles induced magnetic field effect on efficiency enhancement of P3HT:PCBM bulk heterojunction polymer solar cells," Solar Energy Materials and Solar Cells, vol. 95, no. 10, pp. 2880-2885, 2011.

[16] Z. Liu, D. He, Y. Wang, H. Wu, and J. Wang, "Graphene doping of P3HT:PCBM photovoltaic devices," Synthetic Metals, vol. 160, no. 9-10, pp. 1036-1039, 2010.

[17] Y. M. Sung, F. C. Hsu, C. T. Chen, W. F. Su, and Y. F. Chen, "Enhanced photocurrent and stability of inverted polymer/ ZnO-nanorod solar cells by 3-hydroxyflavone additive," Solar Energy Materials and Solar Cells, vol. 98, pp. 103-109, 2012.

[18] Y. Lou, Z. Wang, S. Naka, and H. Okada, "Enhanced shortcircuit current density in poly(3-hexylthiophene) and 1(3-methoxycarbonyl)-propyl-1-phenyl-(6,6)C61 based organic solar cells by doping small molecular perylene," Applied Physics Letters, vol. 99, no. 3, Article ID 033305, 3 pages, 2011.

[19] M. Jørgensen, K. Norrman, and F. C. Krebs, "Stability/degradation of polymer solar cells," Solar Energy Materials and Solar Cells, vol. 92, no. 7, pp. 686-714, 2008.

[20] G. Li, V. Shrotriya, J. Huang et al., "High-efficiency solution processable polymer photovoltaic cells by self-organization of polymer blends," Nature Materials, vol. 4, no. 11, pp. 864-868, 2005.

[21] C. T. Lee and C. H. Lee, "Conversion efficiency improvement mechanisms of polymer solar cells by balance electron-hole mobility using blended P3HT:PCBM:pentacene active layer," Organic Electronics, vol. 14, no. 8, pp. 2046-2050, 2013.

[22] P. N. Murgatroyd, "Theory of space-charge-limited current enhanced by Frenkel effect," Journal of Physics D: Applied Physics, vol. 3, no. 2, article 308, pp. 151-156, 1970.

[23] C. Deibel and V. Dyakonov, "Polymer-fullerene bulk heterojunction solar cells," Reports on Progress in Physics, vol. 73, no. 9, Article ID 096401, 39 pages, 2010.

[24] Y. Terada, N. Takeuchi, S. Yoshida, A. Taninaka, O. Takeuchi, and H. Shigekawa, "Effect of defects buried in pentacene/ alkanethiol self-assembled monolayer/Au film on its electronic properties visualized by scanning tunneling microscopy/spectroscopy," Japanese Journal of Applied Physics, vol. 49, no. 8, Article ID 08LB08, 4 pages, 2010.

[25] C. W. Chu, V. Shrotriya, G. Li, and Y. Yang, “Tuning acceptor energy level for efficient charge collection in copperphthalocyanine-based organic solar cells," Applied Physics Letters, vol. 88, no. 15, Article ID 153504, 3 pages, 2006.

[26] P. Vanlaeke, A. Swinnen, I. Haeldermans et al., "P3HT/PCBM bulk heterojunction solar cells: relation between morphology and electro-optical characteristics," Solar Energy Materials and Solar Cells, vol. 90, no. 14, pp. 2150-2158, 2006.

[27] J. H. Park, J. S. Kim, J. H. Lee, W. H. Lee, and K. Cho, "Effect of annealing solvent solubility on the performance of poly(3hexylthiophene)/ methanofullerene solar cells," Journal of Physical Chemistry C, vol. 113, no. 40, pp. 17579-17584, 2009.

[28] S. Y. Chuang, H. L. Chen, W. H. Lee et al., "Regioregularity effects in the chain orientation and optical anisotropy of composite polymer/fullerene films for high-efficiency, largearea organic solar

cells," Journal of Materials Chemistry, vol. 19, no. 31, pp. 55545560, 2009.

[29] M. Reyes-Reyes, K. Kim, and D. L. Carroll, "High-efficiency photovoltaic devices based on annealed poly(3hexylthiophene) and 1-(3-methoxycarbonyl)-propyl-1- phenyl$(6,6) \mathrm{C}_{61}$ blends," Applied Physics Letters, vol. 87, no. 8, Article ID 083506, 3 pages, 2005.

[30] G. Li, V. Shrotriya, Y. Yao, and Y. Yang, "Investigation of annealing effects and film thickness dependence of polymer solar cells based on poly(3-hexylthiophene)," Journal of Applied Physics, vol. 98, no. 4, Article ID 043704, 5 pages, 2005.

[31] L. Stamenic, E. Smiley, and K. Karim, "Low light conditions modelling for building integrated photovoltaic (BIPV) systems," Solar Energy, vol. 77, no. 1, pp. 37-45, 2004. 

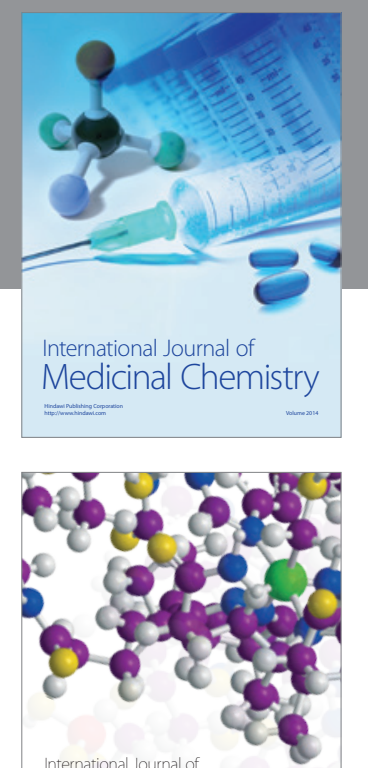

\section{Carbohydrate} Chemistry

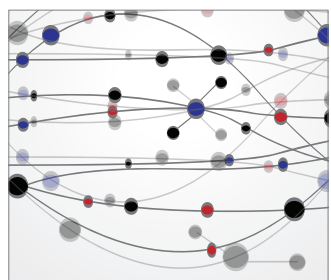

The Scientific World Journal
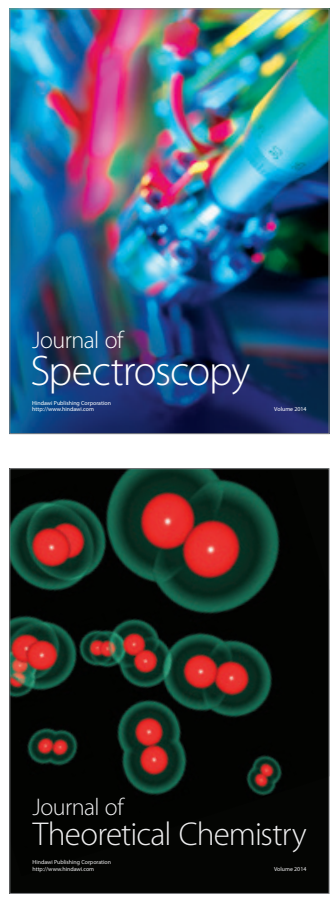
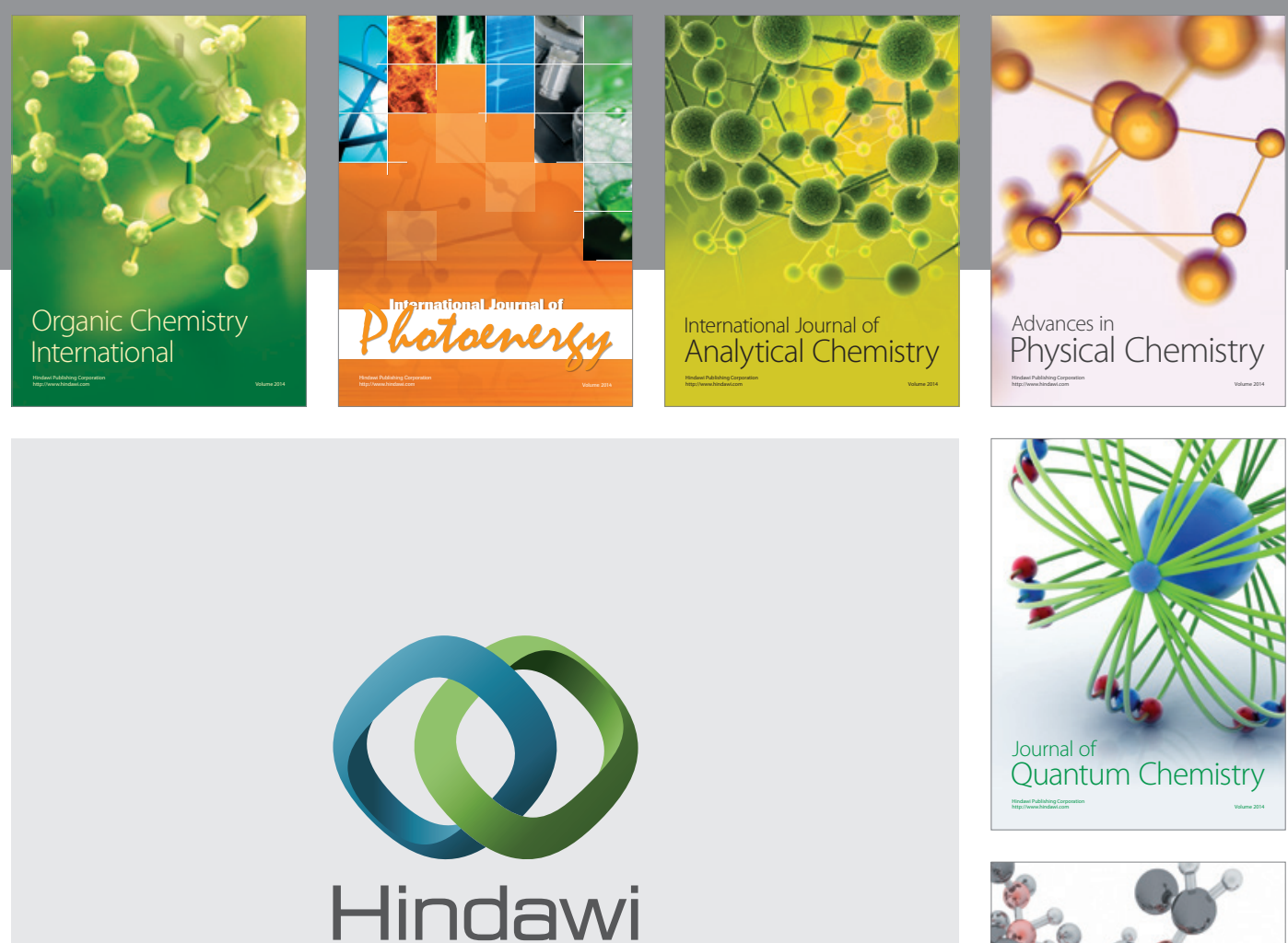

Submit your manuscripts at

http://www.hindawi.com

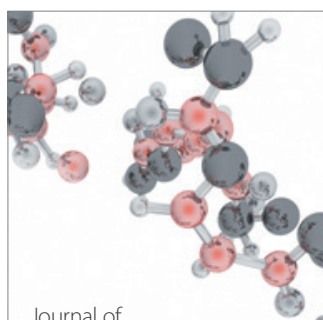

Analytical Methods

in Chemistry

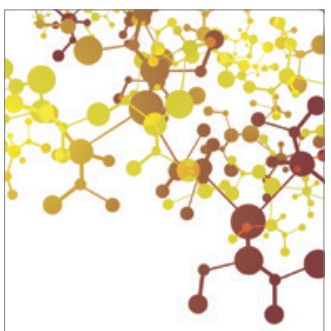

Journal of

Applied Chemistry

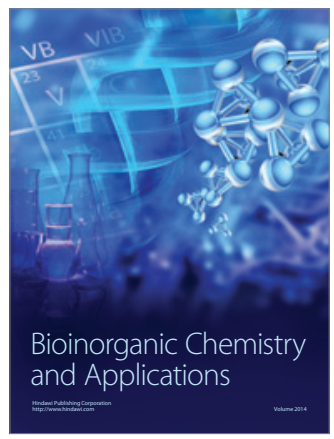

Inorganic Chemistry
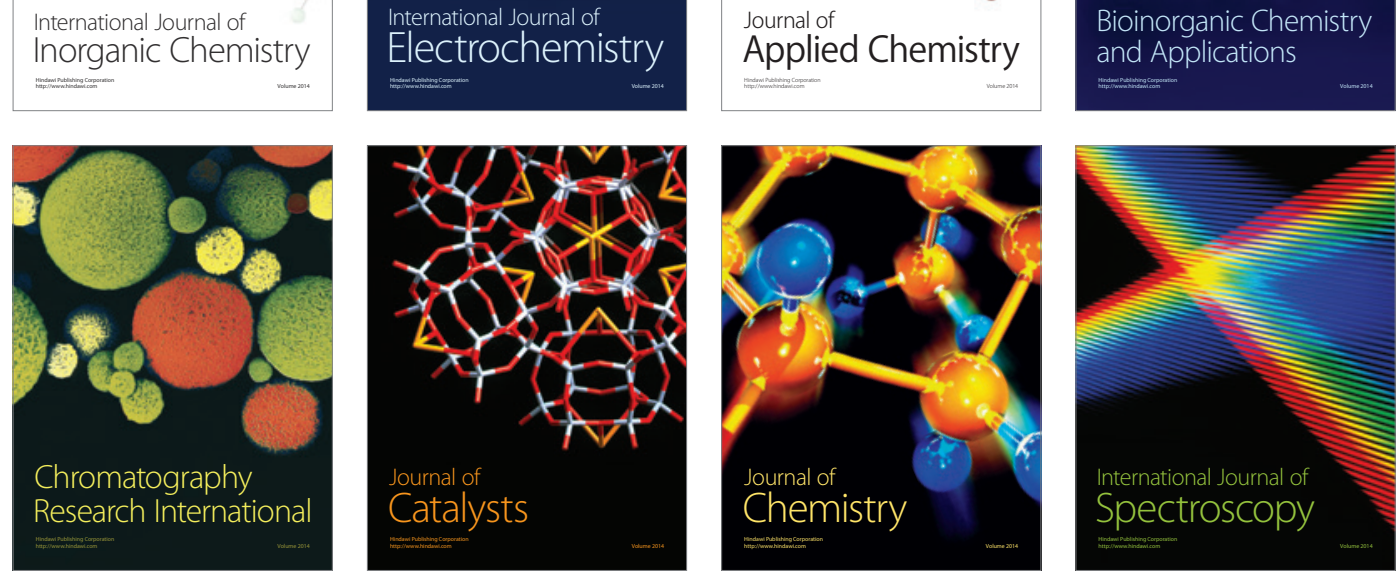